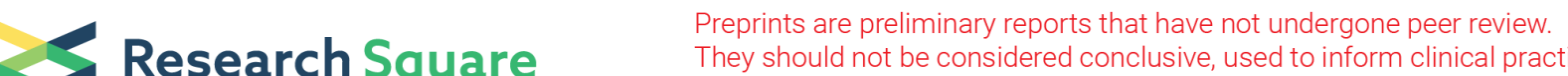 They should not be considered conclusive, used to inform clinical practice, or referenced by the media as validated information.
}

\section{Analysis of Potential Genetic Biomarkers Using Machine Learning Methods and Immune Infiltration Regulatory Mechanisms Underlying Atrial Fibrillation Running Title: Identification of Biomarkers for Af via Machine Learning}

\section{Li-Da Wu}

Wuxi People's Hospital Affiliated to Nanjing Medical University

\section{Feng Li}

Wuxi People's Hospital Affiliated to Nanjing Medical University

Jia-Yi Chen

Wuxi People's Hospital Affiliated to Nanjing Medical University

Jie Zhang

Wuxi People's Hospital Affiliated to Nanjing Medical University

Ling-Ling Qian

Wuxi People's Hospital Affiliated to Nanjing Medical University

Ru-Xing Wang ( $\nabla$ ruxingw@aliyun.com )

Wuxi People's Hospital Affiliated to Nanjing Medical University

\section{Research Article}

Keywords: Atrial fibrillation, Immune infiltration, Biomarker, CIBERSORT, Diagnostic biomarker

Posted Date: December 29th, 2021

DOI: https://doi.org/10.21203/rs.3.rs-1136927/v1

License: (c) (i) This work is licensed under a Creative Commons Attribution 4.0 International License. Read Full License 


\section{Abstract}

Objective: We aimed to screen out biomarkers for atrial fibrillation (AF) based on machine learning methods and evaluate the degree of immune infiltration in AF patients in detail.

Methods: Two datasets (GSE41177 and GSE79768) related to AF in GEO database were included. Differentially expressed genes (DEGs) were screened out using "limma" package. Candidate biomarkers for AF were identified using machine learning methods of the LASSO regression algorithm and SVM-RFE algorithm. Receiver operating characteristic (ROC) curve was employed to assess the diagnostic effectiveness of biomarkers, which was further validated in the GSE14795 dataset. Moreover, we used CIBERSORT to study the proportion of infiltrating immune cells in each sample, and the Spearman method was used to explore the correlation between biomarkers and immune cells.

Results: 129 DEGs were identified, and CYBB, CXCR2, and S100A4 were identified as key biomarkers of AF using LASSO regression and SVM-RFE algorithm, and the diagnostic value was further validated in GSE14795. Immune infiltration analysis indicated that, compared with sinus rhythm (SR), the atrial samples of patients with AF contained a higher T cells gamma delta, neutrophils and mast cells resting, whereas T cells follicular helper were relatively lower. Correlation analysis demonstrated that $C Y B B$, CXCR2, and S100A4 were significantly correlated with the infiltrating immune cells.

Conclusions: In conclusion, this study suggested that CYBB, CXCR2, and S100A4 are key biomarkers correlated with infiltrating immune cells in $\mathrm{AF}$, and infiltrating immune cells play pivotal roles in AF.

\section{Introduction}

As the most common arrhythmia in clinic, patients with atrial fibrillation (AF) have high mortality and morbidity. It is reported that about 1 to $2 \%$ of the population are troubled by $A F$, which contributes to heart failure and cardiogenic embolism [1]. AF could be divided into permanent AF, persistent AF (pAF), long standing pAF and paroxysmal AF based on its duration. Patients with hypertension, obesity, and diabetes etc. frequently develop AF. Molecular mechanisms underlying the development of AF remain unclear yet [2]. Studies have shown that TNF- $a$, CRP and IL -6 are significantly increased in atrial tissues of AF patients and related to outcomes of AF patients [3-5]. Moreover, studies demonstrated the antiinflammatory therapies can significantly reduce AF episodes [6,7]. Yamashita et al. confirmed that, in human AF, adhesion and recruitment of macrophages in heart endocardium promoted inflammatory responses [8]. Similarly, Hohmann et al. demonstrated that the number of CD3-positive T cells in left atrial appendageal are significantly increased in patients with AF [9]. However, the association between infiltrating immune cells and AF still needs to further study.

Medicine is one of the early applications of artificial intelligence (Al), which is gradually changing the way many diseases are diagnosed and treated. Machine learning is an important part of artificial intelligence that using algorithms to identify expression patterns of datasets. Machine learning has been employed in prediction of myocardial infarction, pathological identification and surgical improvement [11]. Moreover, 
machine learning is also a research hotspot and cutting-edge technology in the field of arrhythmia diagnosis and treatment. Han et al. used machine learning algorithms to incorporate clinical signatures of AF and their work provided prognostic value for risk stratification beyond CHA2DS2-VASc for stroke [12]. The diagnosis of atrial fibrillation requires electrocardiogram (ECG) test, but some asymptomatic patients are often difficult to find. Raghunath et al. collected 12 lead ECGs of 430000 patients and predicted new onset atrial fibrillation within 1 year based on deep neural network. It was found that the accuracy of the algorithm reached 0.85 [13]. We firstly conducted a bioinformatics analysis to screen out key differentially expressed genes (DEGs) in AF based on machine learning algorithms. The CIBERSORT algorithm has been asopted to evaluate infiltrating immune cells based on gene expression profiles in various diseases [14-17].

\section{Materials And Methods}

\section{Microarray data}

Three datasets related to pAF (GSE41177, GSE79768 and GSE14975) were downloaded from Gene Expression Omnibus (GEO) database [18] via the "GEO query" package [19]. The above three datasets were all based on GPL570 platform. GSE41177 contained 19 left atrial tissue samples from 16 pAF patients and 3 normal individuals [20]; GSE79768 consisted of 13 left atrial tissue samples from 7 pAF patients and 6 normal individuals [21]; GSE14975 contained 10 left atrial tissue samples from 5 pAF patients and 5 normal individuals [22].

\section{Data processing and DEGs screening}

R was employed to create gene expression matrices of GSE41177, GSE79768 and GSE14975. Log2transformation and background correction were performed on the expression profiles by the "limma" package [23]. GSE41177 and GSE79768 were merged for further analysis, and the GSE14795 dataset was used as the validation cohort. Furthermore, the "SVA" package was used for removing batch effects between the GSE41177 and GSE79768 datasets [24]. "pheatmap" package and "ggplot2" package was adopted to create to heatmap and volcano plot of DEGs.

\section{Enrichment analysis}

To understand the function of DEGs in AF patients, the "clusterProfler" package was used to perform GO and KEGG pathway enrichment analysis [25]. Gene set enrichment analysis (GSEA) was employed to identify terms enriched in pAF patients and normal individuals, respectively.

"c2.cp.kegg.v7.0.symbols.gmt" from the MSigDB database was adopted as the reference [26].

\section{Identification of key DEGs as biomarkers in AF using machine learning methods}

Machine learning methods were adopted to screen out key DEGs as biomarkers in AF. LASSO algorithm is a regression analysis for improving the prediction accuracy [27]. To screen out genes correlated with AF, "glmnet" package was used to carry out LASSO regression algorithm. SVM-RFE is another machine 
learning algorithm, which has been widely used for classification and regression analysis. We also used SVM-RFE algorithm to identify genes with high discriminative power to AF [28].

\section{Diagnostic value of key DEGs as biomarkers in AF}

ROC was established based on the metadata cohort to evaluate the diagnostic effectiveness of biomarkers. Then, we used the AUC value to determine the diagnostic effectiveness, and validated in GSE14795 dataset.

\section{Evaluation of infiltrating immune cells}

CIBERSORT algorithm can be employed to evaluate infiltrating immune cells in patients with AF [17]. A large number of researches have used CIBERSORT to explore the function of immune cells in various diseases, including osteoarthritis [14], high-grade serous ovarian cancer [15] and breast ductal and lobular carcinoma [16]. Proportions of infiltrating immune cells were visualized in R software using "ggplot2" package and "pheatmap" package. Correlation heatmap was created by "corrplot" package to visualize the correlation of infiltrating immune cells. The difference of immune cells infiltration between atrial tissue samples from $\mathrm{AF}$ patients and normal individuals were showed in the violin plot using the "vioplot" package. Then, "ggplot2" package was also adopted to perform PCA analysis based on immune cells infiltration and draw an immune infiltrating heatmap and a two dimensional PCA plot.

\section{Correlation analysis of biomarkers and infiltrating immune cells}

We used the "Spearman" method to explore the correlation between biomarkers and immune infiltration, and then we used the "ggplot2" package was to visualize results.

\section{Results}

\section{Identification of DEGs in AF}

Left atrial tissues from 23 AF patients and 9 normal individuals of GSE41177 and GSE79768 were analyzed. DEGs were identified using the "limma" package. PCA plot clearly indicated that the batch effect between GSE41177 and GSE79768 was successfully removed (Figure 1C). 71 upregulated genes and 58 downregulated genes were obtained in the present study (Figure 1A-B).

\section{Functional correlation analysis}

We performed functional enrichment analysis of DEGs between AF and SR patients based on $\mathrm{GO}$ and KEGG databases (Figure 2A). The biological processes were enriched in neutrophil activation involved in immune response, neutrophil mediated immunity, neutrophil degranulation, neutrophil activation and cell cellular defense response. The relationship between biological processes terms and DEGs was shown in Figure $\mathbf{2 C}$. The enriched cellular components were mainly involved in collagen-containing extracellular 
matrix, secretory granule lumen, cytoplasmic vesicle lumen, vesicle lumen and NADPH oxidase complex. The molecular functions were mainly enriched in RAGE receptor binding, Toll-like receptor binding, calcium-dependent protein binding, superoxide-generating NADPH oxidase activity and long-chain fatty and binding oxidoreductase activity. KEGG pathway analysis shows that the osteoclast differentiation, staphylococcus aureus infection, leukocyte trans-endothelial migration, tight junction and cell adhesion molecules were mostly enriched (Figure 2B). Moreover, GSEA results showed that Hedgehog singling pathway and linoleic acid metabolism were mainly enriched in SR (Figure 3A). The receptor signaling pathway, cell adhesion molecules cams, cytokine cytokine receptor interaction, leukocyte transendothelial migration and natural killer cell mediated cytotoxicity were mainly enriched in AF (Figure 3B).

\section{Identification key DEGs as biomarkers of AF based on machine learning algorithms}

We used two different machine learning algorithms to screen key DEGs as biomarkers of AF. 7 key DEGs were identified using LASSO algorithm in the present study (Figure 4A). Moreover, 40 DEGs was identified as biomarkers via SVM-RFE algorithm (Figure 4B). The three overlapping DEGs (CXCR2, CYBB and S100A4) were ultimately selected (Figure 4C).

\section{Diagnostic effectiveness of biomarkers in AF}

Our results indicated that these three biomarkers screened out by machine learning algorithms also have a favorable diagnostic value in the meta-data cohort, with an AUC of $0.942(95 \% \mathrm{Cl} 0.845-1.000)$ in CYBB, AUC of 0.961 (95\% Cl 0.870-1.000) in CXCR2, and AUC of 0.932 (95\% Cl 0.768-1.000) in S100A4 (Figure 5A-C). In addition, a powerful discrimination ability of AF was confirmed in the GSE14795 dataset with an AUC of 0.880 (95\% Cl $0.600-1.000)$ in $C Y B B$, AUC of 0.760 (95\% Cl $0.400-1.000)$ in CXCR2, and AUC of 0.840 (95\% Cl 0.520-0.912) in S100A4 (Figure 5D-F).

\section{Immune infiltration analysis}

Based on CIBERSORT, we evaluated immune cells infiltration in patients with AF and normal individuals. Figure 6A-B illustrate the proportion of immune cells from 9 SR left atrial tissue samples and $23 \mathrm{AF}$ left atrial tissue samples. As shown in Figure $6 \mathrm{C}$, compared with $\mathrm{SR}$, left atrial tissue samples from AF patients contained higher neutrophils, mast cells resting and $\mathrm{T}$ cells gamma delta, whereas lower $\mathrm{T}$ cells follicular helper. Correlation analysis showed that dendritic cells activated and NK cells resting had the most intense positive relationship with $r=0.62$, B cells naïve and $B$ cells memory had the most obvious negative correlation with $r=-0.51$ (Figure 6D). PCA diagram revealed a distinct group bias and proved that the degree of immune cells infiltration is different between AF patients and normal individuals (Figure S1).

\section{Correlation analysis between CXCR2, CYBB and S100A4 and infiltrating immune cells}

CYBB was positively correlated with $T$ cells gamma delta $(r=0.28, P=0.029)$ and negatively correlated with T cells CD8 $(r=-0.41, P=0.021)$, T cells follicular helper $(r=-0.52, P=0.002)$ (Figure 7A-D). CXCR2 was positively correlated with T cells gamma delta $(r=0.43, P=0.014)$, neutrophils $(r=0.75, P<0.001)$ 
and negatively correlated with macrophages M2 $(r=-0.53, P=0.002)$ (Figure 7E-H). S100A4 was positively correlated with plasma cells $(r=0.45, P=0.009)$ and mast cells resting $(r=0.42, P=0.017)$

(Figure 7I-K).

\section{Discussion}

$\mathrm{AF}$ is the most prevalent cardiac arrhythmia, however, the specific molecular mechanisms of AF still remain unclear. Despite the great improvements has been gained in the field of diagnosis and treatment, AF remains a leading cause of mortality and disability [29]. Drugs for rate control, oral anticoagulants for stroke prevention, antiarrhythmic drug and catheter ablation for conversion are main treatments for $\mathrm{AF}$ patients [30]. But efficacy and safety of these treatment measures are still not well understood.

Meanwhile, many asymptomatic patients, especially with paroxysmal AF, are difficult to find. This is the first study to identify biomarkers of AF associated with immune cells infiltration. Two gene expression datasets from the GEO were merged and conducted an integrated analysis. 129 DEGs were detected using "limma" package. Enrichment analysis showed that these 129 DEGs were significantly correlated with immune and inflammatory responses. The relationship between inflammatory response and AF has been widely studied in the past decades and various regular anti-inflammatory biomarkers were found be related to AF. It has been reported that patients with AF had increased IL-6, IL-8 and TNF-a, meanwhile, these inflammatory markers can predict the outcome of AF ablation [33-37]. High-sensitive C-reactive protein (hsCRP) is also associated with increased risk of AF recurrence following successful electrical cardioversion and catheter ablation [38]. Accumulating studies have demonstrated that the increase of TGF $\beta 1$ in AF patients promote atrial fibrosis, which plays a pivotal role in atrial structural remodeling in AF $[39,40]$. Additionally, the critical role of various immune cells in the pathogenesis of AF has attracted more and more attention, including infiltrating the atrium and secreting several chemokines and cytokines to regulate the microenvironment of the heart [41]. Our GSEA results are also in general agreement with the previous findings that immune cells infiltration participates in the pathogenesis of AF [8-9]. Medicine is the earliest application field of Al. In the past few decades, Al technology, especially machine learning, has made great progress in the diagnosis and treatment of a variety of diseases, including cardiovascular diseases, nervous system diseases and genetic diseases [42]. Therefore, for the first time, we sought to screen out key DEGs between AF and SR patients as biomarkers based on machine learning methods and explore its relationship with immune cells infiltration in AF. Overlapping the results from two machine learning algorithms, CYBB, CXCR2 and S100A4 were identified as key DEGs and biomarkers of AF.

$C Y B B$, also known as NOX2, has been implicated in oxidative stress in various cardiovascular diseases [43]. Pignatelli et al. reported that serum NOX2 can be used as one of the important indicators to predict vascular embolism events in [44]. In animal models, numerous studies have demonstrated that inhibition of NOX2 mediated reactive oxygen species (ROS) prevents atrial remodeling $[45,46]$. In addition, atrial electrical remodeling can also be alleviated by inhibiting NOX2 and oxidative stress [47]. In human AF, NOX2 has also been demonstrated to participate in the atrial structural remodeling and electrophysiological remodeling, and up-regulation of NOX2 is associated with an enhanced risk of AF $[48,49]$. 
The chemokine receptor CXCR2, encoding by CXCR2, belongs to chemokines family, mediates cellular migration of immune cells [50]. The expression level of CXCR2 are tightly regulated during infection and inflammation. CXCR2 is key stimulant of immune cells infiltration and recruitment, especially of neutrophils. Our results of evaluation of 22 subtypes immune cells infiltration showed that neutrophils are significantly elevated in AF patients compared with SR [51]. It is also reported that CXCR2 was involved in atrial monocytes infiltration, which accelerates atrial fibrosis and promotes atrial remodeling. Therefore, blocking CXCR2 may serve as a new therapeutic strategy for AF patients [52]. Moreover, CXCR2 is also a crucial regulator of hypertension. In spontaneously hypertensive rats, Zhang et al. identified that inhibition of CXCR2 could prevent the occurrence of AF [53]. In angiotensin II-induced cardiac atrial fibrillation animal model, CXCR2 has also been proved to participate in immune cells infiltration and mediates cardiac hypertrophy and remodeling through regulation of monocyte [54].

S100A4, also known as fibroblast specific protein 1 (FSP1), is involved in numerous biological processes including cell survival, cell motility, and cell differentiation [55]. Numerous studies have revealed roles of S100A4 in cancer progression, particularly the ability of enhancing metastasis. Nevertheless, S100A4 has been linked to various diseases besides tumor, such as cardiac fibrosis and hypertrophy, kidney fibrosis and pulmonary disease. All of these diseases are involved in inflammatory processes and greatly dependent on tissue remodeling [55]. S100A4 expressed in normal human heart and increased in hypertrophic left ventricles [56-58]. In addition, S100A4 is a key regulator of endothelial mesenchymal transformation (EMT), which is related to immune cell infiltration, making epithelial cells present mesenchymal cell phenotype and ultimately resulting in enhanced migration ability, enhanced anti apoptosis ability and a large number of extracellular matrix components. Recently, studies demonstrated that EMT occurs in the atrium of AF patients and contribute to fibroblast accumulation. Meanwhile, S100A4 also had significant correlations with left atrial dimension in AF patients [59].

We used CIBERSORT to evaluate the degree of infiltrating immune cells in the present study. We found reduced infiltration of $T$ cells follicular helper, as well as increased neutrophils, mast cells resting and $T$ cells gamma delta in AF. Neutrophils represent activated nonspecific inflammation and have been found as markers of inflammation in various diseases. Correlation between inflammatory markers and cardiovascular diseases has been studied widely and the relationship between neutrophils and cardiovascular diseases has been confirmed in the past. It is reported that the level of neutrophils is an independent predictor for the prognosis of acute coronary syndrome [60]. Moreover, accumulating studies have reported that increased neutrophil/lymphocyte ratio is related to the increased risk of AF occurrence $[61,62]$. Mast cells, tissue-specific innate immune cells, present in virtually all body tissues including the heart. Liao et al. reported that mast cells stabilization associated with rescued atrial fibrosis and AF in animal models [63]. Numerous inflammatory mediators secreted by mast cells including IL-1 $\beta$, IL- 6 and TGF- $\beta 1$ also participate in atrial structural remodeling and development of AF [64]. T follicular helper cells has also been reported to function in AF, which might be activated by Toll-like receptor 2 (TLR2) and TLR4 $[65,66]$. We also studied the correlation between $C Y B B, C X C R 2$, S100A4 and infiltrating immune cells. Based on the correlation analysis results, CYBB, CXCR2 and S100A4 appear to play key roles in regulation of immune cells infiltration. 


\section{Conclusions}

We found that $C Y B B, C X C R 2$ and S100A4 may be key biomarkers of AF based on machine learning methods. The immune cells infiltration of patients with AF was measured in detail. Moreover, correlations between $C Y B B, C X C R 2$ and S100A4 and immune cells may play an important role in AF. Further researches for the specific molecular mechanism of these biomarkers and immune cells are required to study.

\section{Declarations}

\section{Data availability statement}

Publicly available datasets were analyzed in this study. All the raw data used in this study are derived from the public GEO data portal (https://www.ncbi.nlm.nih.gov/geo/).

\section{Acknowledgments}

We acknowledge GEO database for providing their platforms and contributors for uploading their meaningful datasets. And we thank all participants involved in studies included in our present study.

\section{Funding}

This work was supported by the Natural Science Foundation of China (81770331) and Research Foundation from Wuxi Health Commission for the Youth囚Q202034『.

Author Contributions: Ru-Xing Wang was involved in the experiment design. Li-Da Wu and Feng Li performed the experiments. Ling-Ling Qian and Jia-Yi Chen analyzed the data. Ru-Xing Wang, Li-Da Wu and Jie Zhang wrote the manuscript. Ling-Ling Qian, Jie Zhang and Jia-Yi Chen edited the manuscript. All authors declare no conflicts of interest. All authors read and approved the final manuscript.

\section{Ethics approval and consent to participate}

Not applicable.

\section{Consent for publication}

Not applicable.

\section{Competing interests}

The authors declare no competing interests.

\section{References}


1. Andrade J, Khairy P, Dobrev D, Nattel S. The clinical profile and pathophysiology of atrial fibrillation: relationships among clinical features, epidemiology, and mechanisms. Circ Res, 2014;114:14531468.

2. Andrade JG, Aguilar M, Atzema C, Bell A, Cairns JA, Cheung CC, et al. The 2020 Canadian cardiovascular society/Canadian heart rhythm society comprehensive guidelines for the management of atrial fibrillation. Can J Cardiol, 2020;36:1847-1948.

3. Chung MK, Martin DO, Sprecher D, Wazni O, Kanderian A, Carnes CA, et al. C-reactive protein elevation in patients with atrial arrhythmias: inflammatory mechanisms and persistence of atrial fibrillation. Circulation, 2001;104:2886-2891.

4. Wu N, Xu B, Xiang Y, Wu L, Zhang Y, Ma X, et al. Association of inflammatory factors with occurrence and recurrence of atrial fibrillation: a meta-analysis. Int J Cardiol, 2013;169:62-72.

5. Marcus GM, Smith LM, Ordovas K, Scheinman MM, Kim AM, Badhwar N, et al. Intracardiac and extracardiac markers of inflammation during atrial fibrillation. Heart Rhythm, 2010;7:149-154.

6. Pfister R, Michels G, Brägelmann J, Sharp SJ, Luben R, Wareham NJ, et al. Plasma vitamin C and risk of hospitalisation with diagnosis of atrial fibrillation in men and women in EPIC-Norfolk prospective study. Int J Cardiol, 2014;177:830-835.

7. Hemilä $\mathrm{H}$, Suonsyrjä T. Vitamin $\mathrm{C}$ for preventing atrial fibrillation in high risk patients: a systematic review and meta-analysis. BMC Cardiovasc Disord, 2017;17:49.

8. Yamashita T, Sekiguchi A, Iwasaki YK, Date T, Sagara K, Tanabe H, et al. Recruitment of immune cells across atrial endocardium in human atrial fibrillation. Circ J, 2010;74:262-270.

9. Hohmann C, Pfister R, Mollenhauer M, Adler C, Kozlowski J, Wodarz A, et al. Inflammatory cell infiltration in left atrial appendageal tissues of patients with atrial fibrillation and sinus rhythm. Sci Rep, 2020;10:1685.

10. Yu KH, Beam AL, Kohane IS. Artificial intelligence in healthcare. Nat Biomed Eng, 2018;2(10):719731.

11. Rajkomar A, Dean J, Kohane I. Machine learning in medicine. N Engl J Med, 2019;380(14): 13471358.

12. Han L, Askari M, Altman RB, Schmitt SK, Fan J, Bentley JP, et al. Atrial fibrillation burden signature and near-term prediction of stroke: a machine learning analysis. Circ Cardiovasc Qual Outcomes, 2019;12(10):e005595.

13. Raghunath S, Pfeifer JM, Ulloa-Cerna AE, Nemani A, Carbonati T, Jing L, et al. Deep neural networks can predict new-onset atrial fibrillation from the 12-lead ECG and help identify those at risk of atrial fibrillation-related stroke. Circulation, 2021;143(13): 1287-1298.

14. Deng YJ, Ren EH, Yuan WH, Zhang GZ, Wu ZL, Xie QQ, et al. GRB10 and E2F3 as diagnostic markers of osteoarthritis and their correlation with immune infiltration. Diagnostics (Basel), 2020;10(3):171.

15. Liu R, Hu R, Zeng Y, Zhang W, Zhou H-H. Tumour immune cell infiltration and survival after platinumbased chemotherapy in high-grade serous ovarian cancer subtypes: a gene expression-based computational study. EBioMedicine, 2020;51:102602. 
16. Zhang B, Wang Q, Fu C, Jiang C, Ma S. Exploration of the immune-related signature and immune infiltration analysis for breast ductal and lobular carcinoma. Ann Transl Med, 2019;7(23):730.

17. Newman AM, Liu CL, Green MR, Gentles AJ, Feng W, Xu Y, et al. Robust enumeration of cell subsets from tissue expression profiles. Nature Methods, 2015;12:453-457.

18. Barrett T, Wilhite SE, Ledoux P, Evangelista C, Kim IF, Tomashevsky M, et al. NCBI GEO: Archive for functional genomics data sets-update. Nucleic Acids Res, 2013;41:991-995.

19. Davis S, Meltzer PS. GEOquery: a bridge between the Gene Expression Omnibus (GEO) and BioConductor. Bioinformatics, 2007;23(14):1846-1847.

20. Yeh YH, Kuo CT, Lee YS, Lin YM, Nattel S, Tsai FC, et al. Region-specific gene expression profiles in the left atria of patients with valvular atrial fibrillation. Heart Rhythm, 2013;10(3):383-391.

21. Tsai FC, Lin YC, Chang SH, Chang GJ, Hsu YJ, Lin YM, et al. Differential left-to-right atria gene expression ratio in human sinus rhythm and atrial fibrillation: Implications for arrhythmogenesis and thrombogenesis. Int J Cardiol, 2016;222:104-112.

22. Adam O, Lavall D, Theobald K, Hohl M, Grube M, Ameling S, et al. Rac1-induced connective tissue growth factor regulates connexin 43 and $\mathrm{N}$-cadherin expression in atrial fibrillation. J Am Coll Cardiol, 2010;55(5):469-480.

23. Ritchie ME, Phipson B, Wu D, Hu Y, Law CW, Shi W, et al. limma powers differential expression analyses for RNA-sequencing and microarray studies. Nucleic Acids Res, 2015;43(7):e47.

24. Leek JT, Johnson WE, Parker HS, Jaffe AE, Storey JD. The sva package for removing batch effects and other unwanted variation in high-throughput experiments. Bioinformatics, 2012;28:882-883.

25. Wu T, Hu E, Xu S, Chen M, Guo P, Dai Z, et al. clusterProfiler 4.0: A universal enrichment tool for interpreting omics data. Innovation (N Y), 2021, 2(3):100141.

26. Powers RK, Goodspeed A, Pielke-Lombardo H, Tan AC, Costello JC. GSEA-InContext: identifying novel and common patterns in expression experiments. Bioinformatics, 2018;34(13):i555-i564.

27. Wang H, Lengerich BJ, Aragam B, Xing EP. Precision Lasso: accounting for correlations and linear dependencies in high-dimensional genomic data. Bioinformatics, 2019;35(7):1181-1187.

28. Tang J, Wang Y, Luo Y, Fu J, Zhang Y, Li Y, et al. Computational advances of tumor marker selection and sample classification in cancer proteomics. Comput Struct Biotechnol J, 2020;18:2012-2025.

29. Chugh SS, Havmoeller R, Narayanan K, Singh D, Rienstra M, Benjamin EJ, et al. Worldwide epidemiology of atrial fibrillation: a Global Burden of Disease 2010 Study. Circulation, 2014;129:837-847.

30. January CT, Wann LS, Calkins H, Chen LY, Cigarroa JE, Cleveland JC Jr, et al. 2019 AHA/ACC/HRS focused update of the 2014 AHA/ACC/HRS guideline for the management of patients with atrial fibrillation: a report of the American college of cardiology/American heart association task force on clinical practice guidelines and the heart rhythm society in collaboration with the society of thoracic surgeons. Circulation 2019;140:e125-151. 
31. Luan Y, Guo Y, Li S, et al. Interleukin-18 among atrial fibrillation patients in the absence of structural heart disease. Europace, 2010;12:1713-1718.

32. Qu YC, Du YM, Wu SL, et al. Activated nuclear factor kappaB and increased tumor necrosis factoralpha in atrial tissue of atrial fibrillation. Scand Cardiovasc J, 2009;43:292-297.

33. Psychari SN, Apostolou TS, Sinos L, et al. Relation of elevated C-reactive protein and interleukin-6 levels to left atrial size and duration of episodes in patients with atrial fibrillation. Am J Cardiol, 2005;95:764-767.

34. Liuba I, Ahlmroth $\mathrm{H}$, Jonasson $\mathrm{L}$, et al. Source of inflammatory markers in patients with atrial fibrillation. Europace, 2008;10:848-853.

35. Marcus GM, Whooley MA, Glidden DV, et al. Interleukin-6 and atrial fibrillation in patients with coronary artery disease: data from the Heart and Soul Study. Am Heart J, 2008;155:303-309.

36. Kornej J, Reinhardt C, Kosiuk J, Arya A, Hindricks G, Adams V, et al. Response of high-sensitive Creactive protein to catheter ablation of atrial fibrillation and its relation with rhythm outcome. PLoS One, 2012;7:e44165.

37. Kunamalla A, Ng J, Parini V, Yoo S, McGee KA, Tomson TT, et al. Constitutive expression of a dominant-negative TGF- $\beta$ type II receptor in the posterior left TGF- $\beta$ type II receptor in the posterior left atrium leads to beneficial remodeling of atrial fibrillation substrate. Circ Res, 2016;119:69-82.

38. Teodorescu P, Pasca S, Jurj A, Gafencu G, Joelsson JP, Selicean S, et al. Transforming growth factor$\beta$-mediated CD44/STAT3 signaling contributes to the development of atrial fibrosis and fibrillation. Basic Res Cardiol, 2017;112:58.

39. Guo Y, Lip GY, Apostolakis S. Inflammation in atrial fibrillation. J Am Coll Cardiol, 2012;60:22632270.

40. Deo RC. Machine Learning in Medicine. Circulation, 2015;132(20):1920-1930.

41. Zhang M, Perino A, Ghigo A, Hirsch E, Shah AM. NADPH oxidases in heart failure: poachers or gamekeepers? Antioxid Redox Signal, 2013;18(9):1024-1041.

42. Pignatelli P, Pastori D, Carnevale R, Farcomeni A, Cangemi R, Nocella C, et al. Serum NOX2 and urinary isoprostanes predict vascular events in patients with atrial fibrillation. Thromb Haemost, 2015;113(3):617-624.

43. Saito S, Teshima Y, Fukui A, Kondo H, Nishio S, Nakagawa M, et al. Glucose fluctuations increase the incidence of atrial fibrillation in diabetic rats. Cardiovasc Res, 2014;104(1):5-14.

44. Chen WJ, Chang SH, Chan YH, Lee JL, Lai YJ, Chang GJ, et al. Tachycardia-induced CD44/NOX4 signaling is involved in the development of atrial remodeling. J Mol Cell Cardiol, 2019;135:67-78.

45. Lu G, Li J, Zhai Y, Li Q, Xie D, Zhang J, et al. Spironolactone suppresses aldosterone-induced Kv1.5 expression by attenuating mineralocorticoid receptor-Nox1/2/4-mediated ROS generation in neonatal rat atrial myocytes. Biochem Biophys Res Commun, 2019;520(2):379-384.

46. Violi F, Carnevale R, Calvieri C, Nocella C, Falcone M, Farcomeni A, et al. Nox2 up-regulation is associated with an enhanced risk of atrial fibrillation in patients with pneumonia. Thorax, 
2015;70(10):961-966.

47. Kim YM, Guzik TJ, Zhang YH, Zhang MH, Kattach H, Ratnatunga C, et al. A myocardial Nox2 containing $\mathrm{NAD}(\mathrm{P}) \mathrm{H}$ oxidase contributes to oxidative stress in human atrial fibrillation. Circ Res, 2005;97(7):629-636.

48. Planagumà $A$, Domènech $T$, Pont $M$, Calama $E$, García-González $V$, López $R$, et al. Combined anti CXC receptors 1 and 2 therapy is a promising anti-inflammatory treatment for respiratory diseases by reducing neutrophil migration and activation. Pulm Pharmacol Ther, 2015;34:37-45.

49. Zhang X, Guo R, Kambara H, Ma F, Luo HR. The role of CXCR2 in acute inflammatory responses and its antagonists as anti-inflammatory therapeutics. Curr Opin Hematol, 2019;26(1):28-33.

50. Zhang YL, Cao HJ, Han X, Teng F, Chen C, Yang J, et al. Chemokine receptor CXCR-2 initiates atrial fibrillation by triggering monocyte mobilization in mice. Hypertension, 2020;76(2):381-392.

51. Zhang YL, Cao HJ, Han X, Teng F, Chen C, Yang J, et al. Chemokine receptor CXCR-2 initiates atrial fibrillation by triggering monocyte mobilization in mice. Hypertension, 2020;76(2):381-392.

52. Wang L, Zhang YL, Lin QY, Liu Y, Guan XM, Ma XL, et al. CXCL1-CXCR2 axis mediates angiotensin IIinduced cardiac hypertrophy and remodelling through regulation of monocyte infiltration. Eur Heart $\mathrm{J}$, 2018;39: 1818-1831.

53. Schneider M, Hansen JL, Sheikh SP. S100A4: a common mediator of epithelial-mesenchymal transition, fibrosis and regeneration in diseases? J Mol Med (Berl), 2008;86(5):507-522.

54. Lawson WE, Polosukhin VV, Zoia O, Stathopoulos GT, Han W, Plieth D, Loyd JE, Neilson EG, Blackwell TS. Characterization of fibroblast-specific protein 1 in pulmonary fibrosis. Am J Respir Crit Care Med, 2005;171:899-907.

55. Schneider M, Kostin S, Strom CC, Aplin M, Lyngbaek S, Theilade J, et al. S100A4 is upregulated in injured myocardium and promotes growth and survival of cardiac myocytes. Cardiovasc Res, 2007;75:40-50.

56. Strøm CC, Kruhøffer M, Knudsen S, Stensgaard-Hansen F, Jonassen TEN, Ørntoft TF, Haunsø S, et al. Identification of a core set of genes that signifies pathways underlying cardiac hypertrophy. Comp Funct Genomics, 2004;5:459-470.

57. Kato T, Sekiguchi A, Sagara K, Tanabe H, Takamura M, Kaneko S, et al. Endothelial-mesenchymal transition in human atrial fibrillation. J Cardiol, 2017;69(5):706-711.

58. Bhat T, Teli S, Rijal J, Bhat H, Raza M, Khoueiry G, Neutrophil to lymphocyte ratio and cardiovascular diseases: a review. Expert Rev Cardiovasc Ther, 2013;11(1):55-59. doi: 10.1586/erc.12.159.

59. Shao Q, Chen K, Rha SW, Lim HE, Li G, Liu T. Usefulness of neutrophil/lymphocyte ratio as a predictor of atrial fibrillation: a meta-analysis. Arch Med Res, 2015;46(3):199-206.

60. Paquissi FC. The predictive role of inflammatory biomarkers in atrial fibrillation as seen through neutrophil-lymphocyte ratio mirror. J Biomark, 2016;2016:8160393.

61. Liao C, Akazawa H, Tamagawa M, Ito K, Y asuda N, Kudo Y, et al. Cardiac mast cells cause atrial fibrillation through PDGF-A-mediated fibrosis in pressure-overloaded mouse hearts. J Clin Invest, 
$2010,120: 242-253$.

62. Legere SA, Haidl ID, Légaré JF, Marshall JS. Mast cells in cardiac fibrosis: new insights suggest opportunities for intervention. Front Immunol, 2019, 28;10:580.

63. Hu YF, Chen YJ, Lin YJ, Chen SA. Infammation and the pathogenesis of atrial fibrillation. Nat Rev Cardiol. 2015;12(4):230-243.

64. Kared H, Camous X, Larbi A. T cells and their cytokines in persistent stimulation of the immune system. Curr Opin Immunol, 2014;29:79-85.

\section{Figures}

A

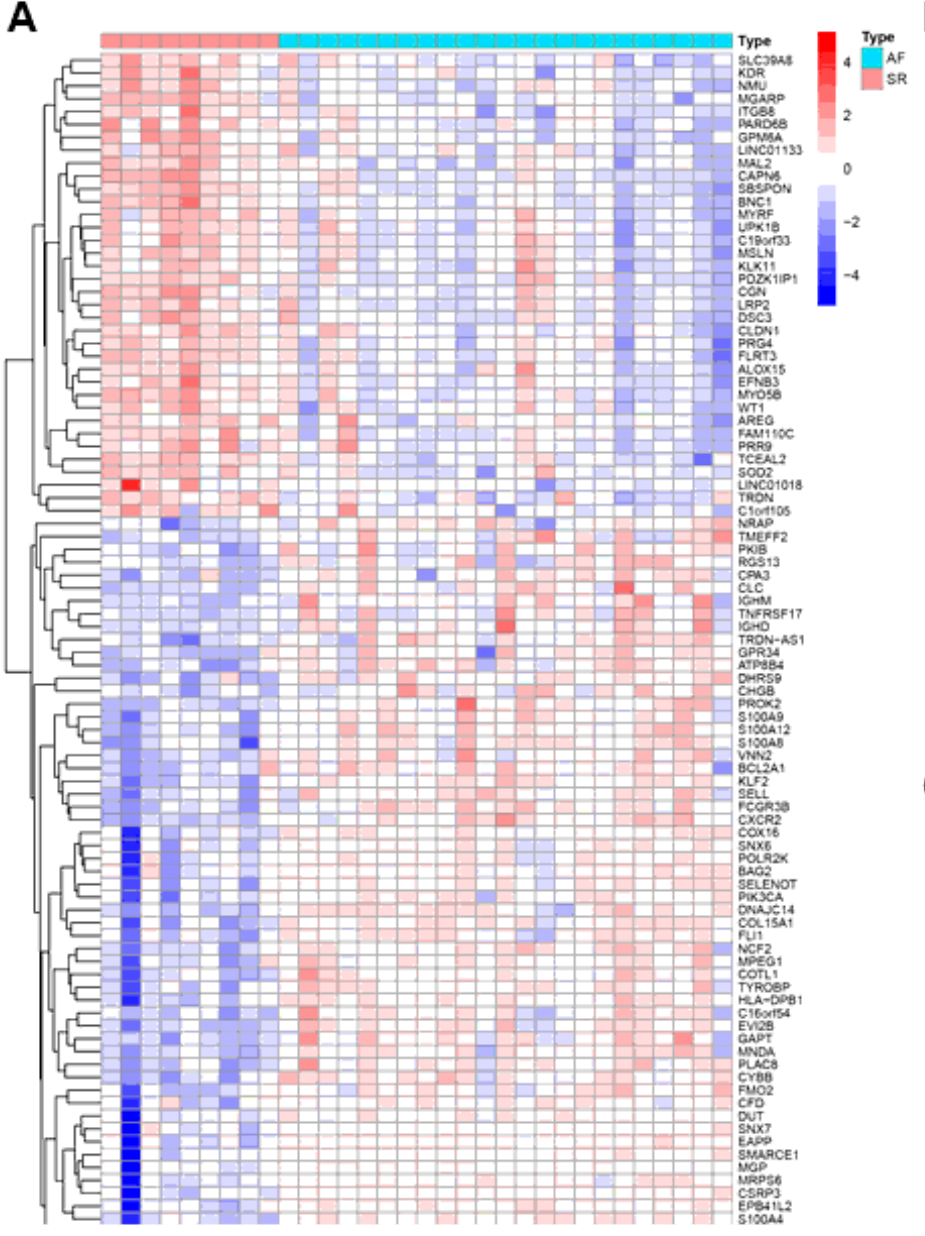

B

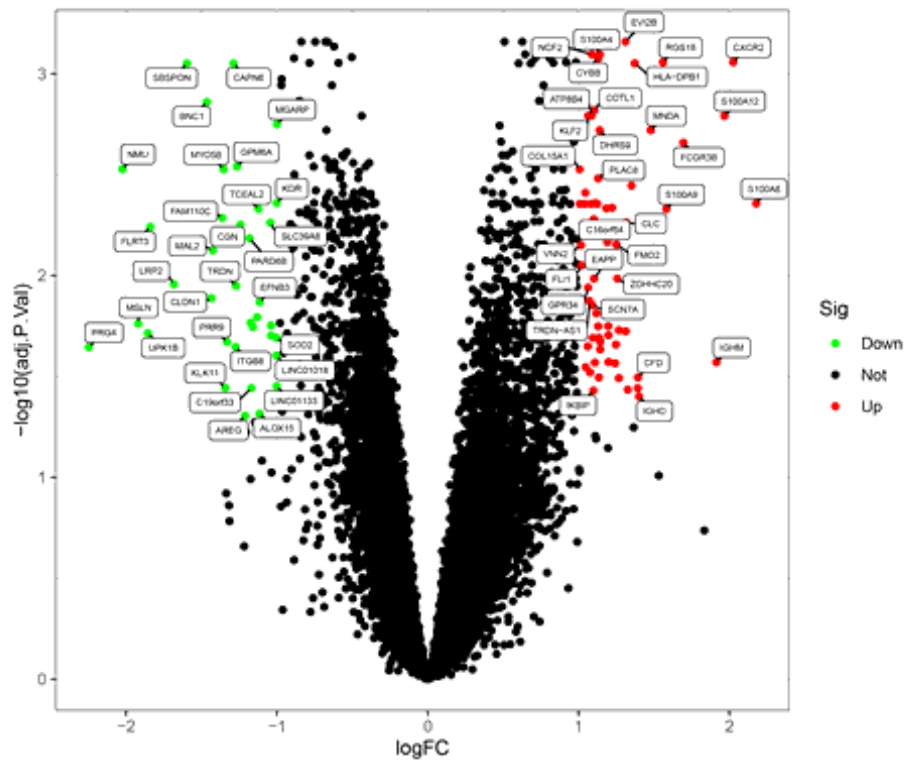

C

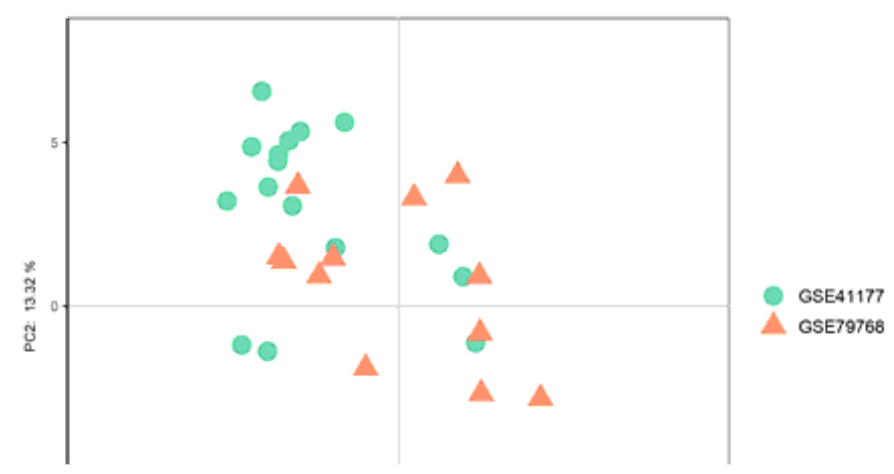

Figure 1

Identification of DEGs between AF and SR atrial tissue samples. A Heatmap visualization of the top 100 DEGs between AF and SR atrial tissue samples. B Volcano plot visualization of DEGs between AF and SR 

GSE41177 and GSE79768. AF, atrial fibrillation; SR, sinus rhythm; DEGs, differentially expressed genes.

A

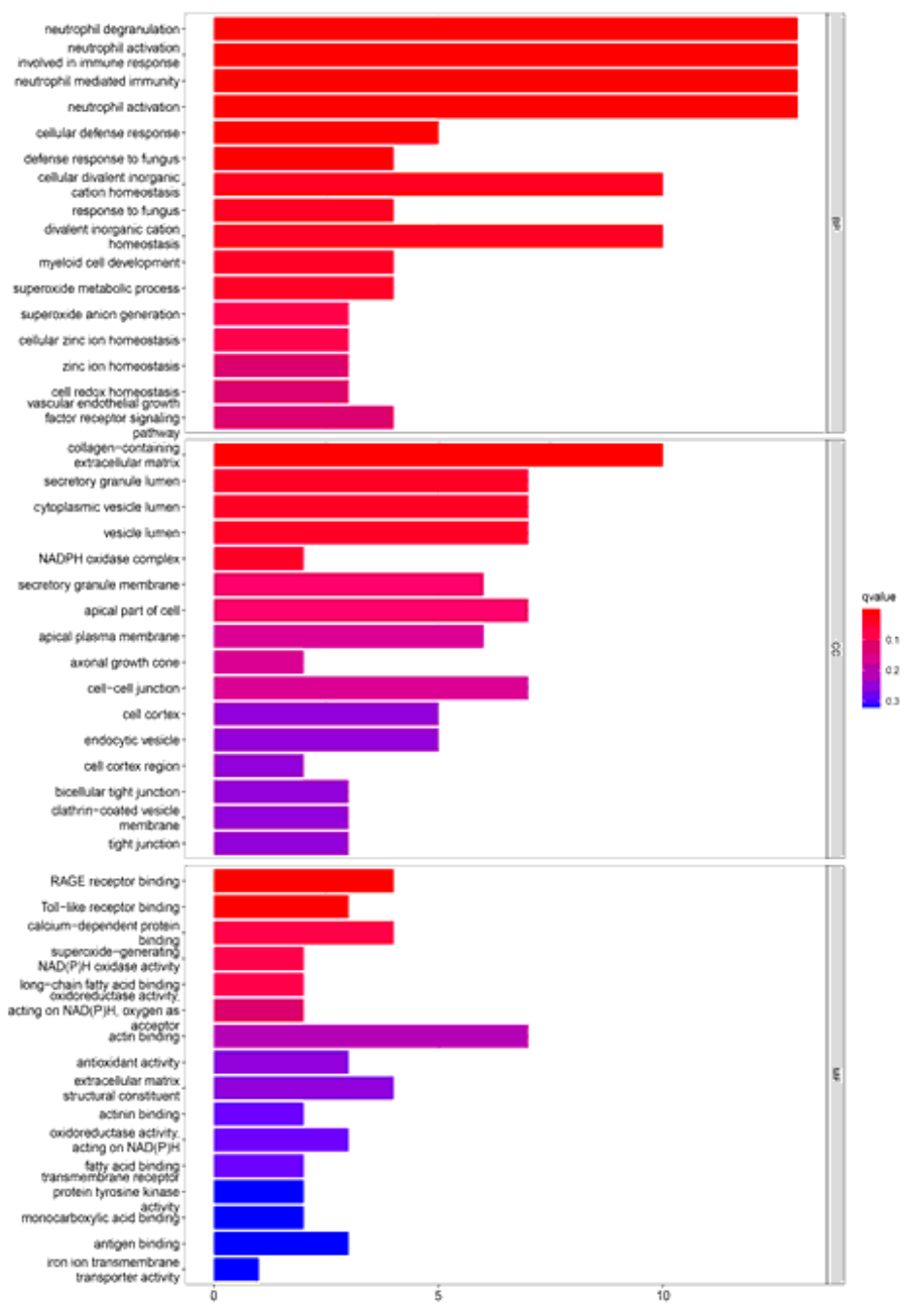

B

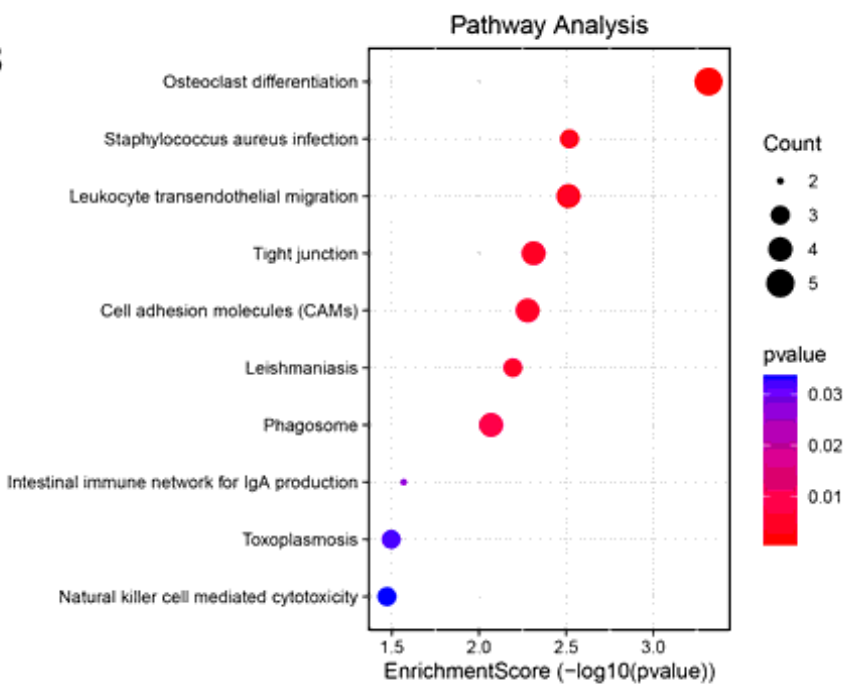

C

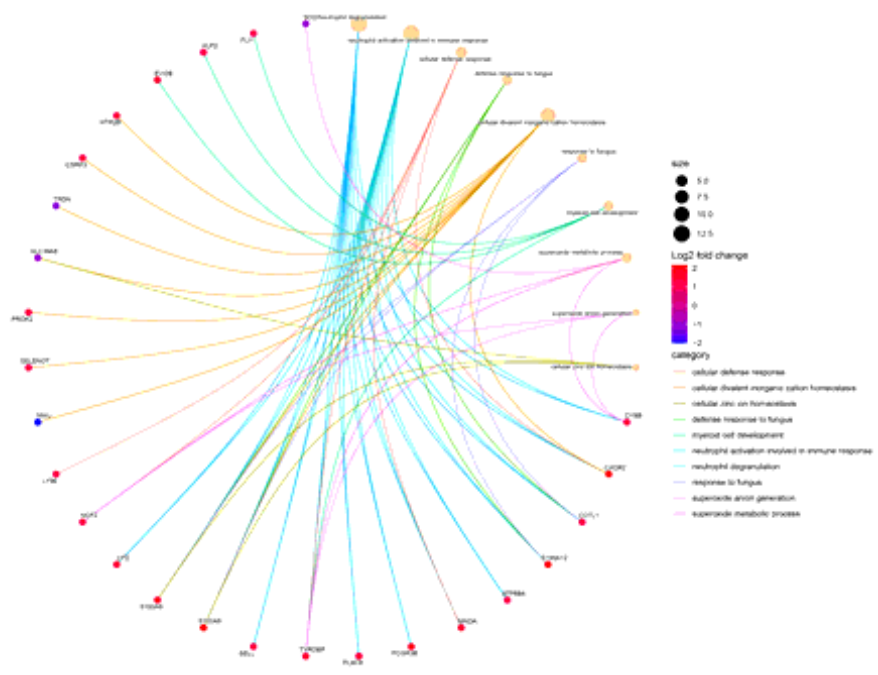

Figure 2

Enrichment analysis of DEGs between AF and SR atrial tissue samples via $\mathrm{GO}$ and KEGG database. A Gene ontology enrichment analysis of DEGs. B KEGG enrichment analysis of DEGs. C Cord diagram shows the relationship between key DEGs and most enriched biological processes. AF, atrial fibrillation; $\mathrm{SR}$, sinus rhythm; DEGs, differentially expressed genes.

\section{Figure 3}

GSEA enrichment analysis of DEGs between AF and SR atrial tissue samples. A GSEA enrichment analysis results in SR patients. B GSEA enrichment analysis results in AF patients. AF, atrial fibrillation; SR, sinus rhythm; GSEA, gene set enrichment analysis; DEGs, differentially expressed genes. 


\section{Figure 4}

Identification of biomarker candidates for AF based on machine learning algorithms. A Biomarkers selection via LASSO algorithm. B Biomarkers selection via SVM-RFE algorithm. C Venn plot of the overlapping genes identified by the LASSO algorithm and SVM-RFE algorithm. AF, atrial fibrillation; LASSO, least absolute shrinkage and selection operator model; SVM-RFE, support vector machinerecursive feature elimination model.

\section{Figure 5}

Evaluation of the diagnostic effectiveness of the three biomarkers. A-C ROC curve of $C Y B B, C X C R 2$ and S100A4 in the metadata cohort of GSE41177 and GSE79768; D-E ROC curve of CYBB, CXCR2 and S100A4 in the validation data of GSE14795. ROC, receiver operating characteristic.

\section{Figure 6}

Evaluation and visualization of immune cells infiltration in AF and SR atrial tissue samples. A The proportion of infiltrating immune cells in AF and SR atrial tissue samples. B Heatmap of infiltrating immune cells in AF and SR atrial tissue samples. C The difference of 22 subpopulations of immune cells between AF and SR atrial tissue samples. D Correlation heatmap shows the correlation between 22 immune cell subpopulations. AF, atrial fibrillation; SR, sinus rhythm.

\section{Figure 7}

Correlations between CYBB, CXCR2, S100A4 and infiltrating immune cells in AF. A-D Correlation between CYBB and infiltrating immune cells in AF. E-H Correlation between CXCR2 and infiltrating immune cells in AF. I-K Correlation between S100A4 and infiltrating immune cells in AF. AF, atrial fibrillation.

\section{Supplementary Files}

This is a list of supplementary files associated with this preprint. Click to download.

- FigS1.tif 